\title{
Pipeline Robot Localization Technique Based on Inductive Coupling
}

\author{
Zhigang Li ${ }^{1, a}$, Yujia Liu' ${ }^{2, b}$, Qingdong Ding ${ }^{2, c}$ and Wei Xue ${ }^{2, d}$ \\ ${ }^{1}$ College of Information and Communication Engineering of Harbin Engineering University, Harbin \\ 150001, China; \\ ${ }^{2}$ College of Information and Communication Engineering of Harbin Engineering University, Harbin \\ 150001, China. \\ alizhigang@hrbeu.edu.cn, bliuyujia@hrbeu.edu.cn, 'dingqingdong@hrbeu.edu.cn, \\ dxuewei@hrbeu.edu.cn
}

Keywords: Inductive Coupling; Magnetic Antenna; Matlab; FPGA; Positioning.

\begin{abstract}
The mathematical model of the magnetic field distribution of the magnetic dipole antenna is established. The simulation process is realized by using the Matlab software, which provides a preliminary theoretical basis for the transmitting and receiving positioning of the magnetic antenna. The magnetic field distribution and strength of the magnetic dipole antenna are measured and the simulation results are compared with the theoretical model. The frequency and power of the magnetic dipole antenna are completed by the hardware platform of physical experiment hardware. Parameter optimization design. On this basis, the principle prototype development of pipeline robot positioning system is completed.
\end{abstract}

\section{Introduction}

Currently, more state detection is carried out by intelligent robots into the pipeline and run along the pipeline. In the oil and gas pipeline in the defect detection and pigging operations, due to the greater degree of pipe line bending, the tube too much sediment will happen occasionally detectors and pig loss or stuck and other accidents, we must accurately locate and find back to the lost equipment, which is critical for the maintenance of the pipeline. At present, the technology of tracking and locating the detectors in the pipeline mainly includes traditional wheel positioning, data fusion positioning of multi-mileage wheels, multi-sensor integrated positioning, fixed magnetic marker positioning, visual sensor positioning and radioactive ray positioning technology. For deep buried underground or sea water pipelines, the use of ultra-low frequency electromagnetic field based positioning method, compared with the traditional positioning method using $\gamma$-ray, very low frequency electromagnetic signal does not exist radioactive, will not damage the marine ecological environment and damage to the operator's health.

\section{Simulation of Magnetic Field Distribution of Magnetic Dipole}

Extremely low frequency signals refer to electromagnetic signals with frequencies between 3 and 30 Hz. They can penetrate seawater, rocks and even metals, and are widely used in the fields of resource exploration, earthquake prediction, drilling telemetry and submarine communication. Tracking and Location of Intelligent Robots in. Here the use of magnetic dipole as the model of the antenna system to achieve the positioning of the pipeline robot. 
The magnetic dipole is a circular current, the magnetic fundamental oscillator is the ideal magnetic dipole, any current carrying thin wire loop can be seen as a magnetic basic oscillator, shown in Figure 1 for the magnetic basic oscillator model. It is a small circle in the $\mathrm{x}-\mathrm{y}$ plane. The diameter of the wire is negligible. The current on the wire is approximated by a line current, and the amplitude and phase of the current in the ring are equal everywhere. Ring on the time-harmonic current, $\mathrm{i}=I_{m} \cos (\omega t+$ $\varphi$ ), so the complex number is expressed as $\mathrm{I}=I_{m} e^{j \varphi}$. The magnetic dipole moment $\mathrm{M}$ is defined as: $\mathrm{M}=\mathrm{IS}$, where $\mathrm{I}$ is a complex number of current, $\mathrm{S}$ is the direction of the circuit area, if the right hand four fingers in line with the current direction, the direction of the thumb S direction. In Fig. 1, the direction of $\mathrm{S}$ is the positive direction of the coordinate axis $\mathrm{z}$. Magnetic dipole in the axial $\mathrm{n}$ at any point on the magnetic induction of intensity:

$$
\mathrm{B}=\frac{\mu_{0}}{2 \pi} \frac{M}{r^{3}}
$$

In the lateral on any point of the magnetic induction intensity is:

$$
\mathrm{B}=\frac{\mu_{0}}{4 \pi} \frac{M}{r^{3}}
$$

Where $r$ is the distance from the center of the magnetic dipole to the spatial point. It can be preliminarily determined that the magnetic induction of a magnetic dipole at a certain point in space is proportional to its magnetic moment and inversely proportional to the third power of the distance from the center of the magnetic dipole.

All manuscripts must be in English, also the table and figure texts, otherwise we cannot publish your paper. Please keep a second copy of your manuscript in your office. When receiving the paper, we assume that the corresponding authors grant us the copyright to use the paper for the book or journal in question. Should authors use tables or figures from other Publications, they must ask the corresponding publishers to grant them the right to publish this material in their paper.

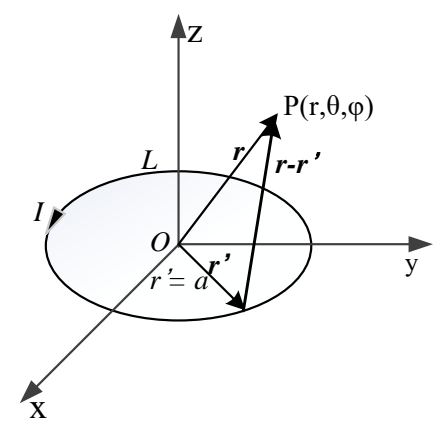

Fig. 1 Magnetic fundamental oscillator

In an infinitely large conductive medium, the magnetic dipole-excited electromagnetic field components are expressed as $(\sigma \gg \omega \varepsilon)$ :

$$
\begin{gathered}
H_{r}=\frac{m \cos \theta}{2 \pi r^{3}}(1+\beta r+j \beta r) e^{-\beta r} e^{-j \beta r} \\
H_{\varnothing}=\frac{m \sin \theta}{4 \pi r^{3}}\left[1+\beta r+j\left(\beta r+2 \beta^{2} r^{2}\right)\right] e^{-\beta r} e^{-j \beta r}
\end{gathered}
$$

Through the Matlab simulation can be seen magnetic field intensity $\mathrm{H}$ with three-dimensional simulation map receiving distance and $\theta$ changes. 


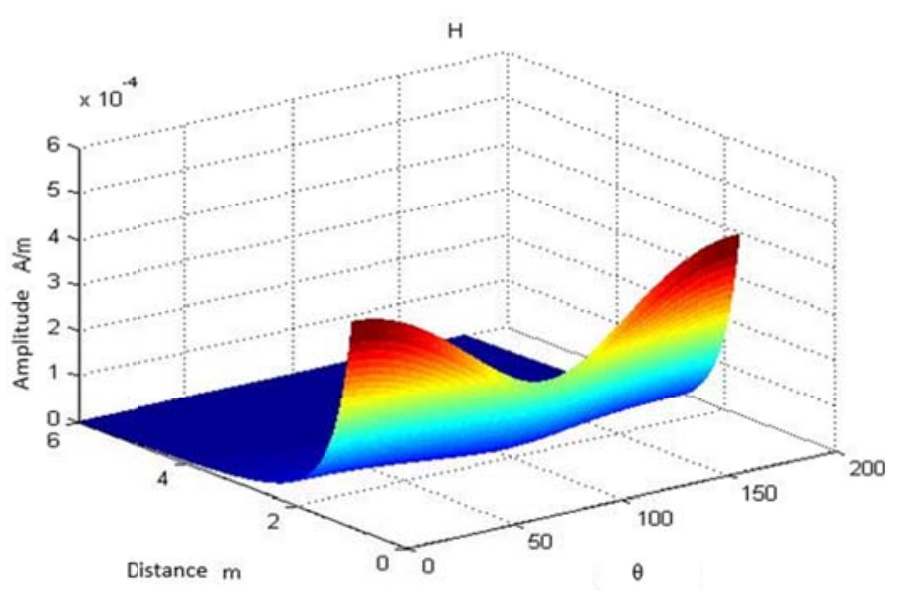

Fig. 2 Simulation Results

It can be seen that the magnetic field intensity $\mathrm{H}$ has the maximum value in the $\mathrm{Z}$ axis direction, and with the increase of the distance, the intensity of the magnetic field decays very quickly. In the horizontal direction, the magnetic field intensity has the characteristic of symmetry.

\section{Positioning Scheme}

According to the law of magnetic field variation, the magnetic antenna can be installed on the pipeline robot parallel to the pipe axial direction. According to the change law of $B_{x}$ with $\mathrm{z}$ easy to know $\left|B_{x}\right|$ with $\mathrm{z}$ will show a bimodal distribution, as shown in Figure 3 shows the minimum value between the two signals is the location of the location of the source. Therefore, in the positioning process, the receiving antenna generates the amplitude signal through the change of the magnetic field in the direction perpendicular to the transmitting antenna. As the relative position of the receiving antenna and the transmitting antenna changes, the signal strength on the receiving antenna changes accordingly. By detecting such signal strength change and find the position of the minimum point between the bimodal point that is the location of the emission source, the pipe robot can achieve the location of the tube outside the tube positioning.

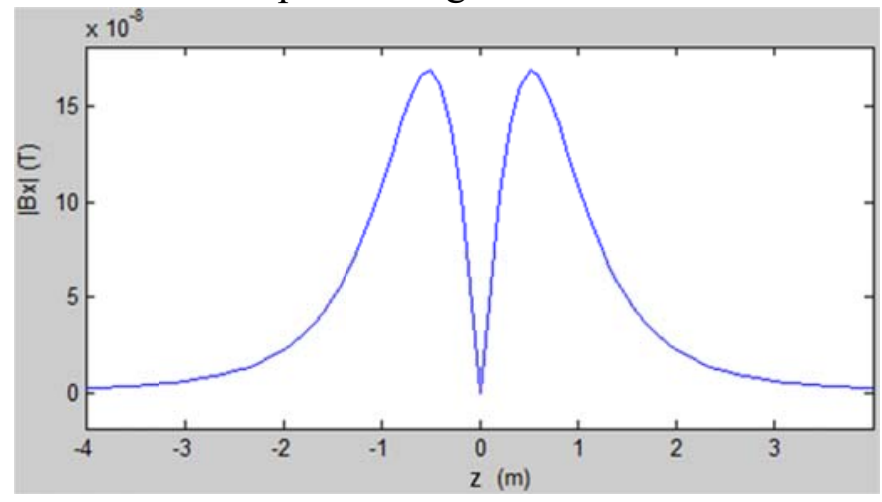

Fig. $3\left|B_{x}\right|$ changes with $\mathrm{z}$

Therefore, if a single magnetic antenna is used for receiving and positioning, when the receiving antenna and the transmitting antenna are in a vertical state, the receiving antenna can determine the emission in a rest state by measuring the spatial distribution of the reconstructed electromagnetic field according to the change of $\left|B_{x}\right|$. If the receiver antenna is in the position $\mathrm{A}$ in the Figure 4, it can be detected that the maximum value of the receiver antenna can be obtained when the receiving antenna is in parallel with the transmitter in the pig. Magnetic inductive strength, and in its left and right sides near the symmetrical position of each have a minimum. 


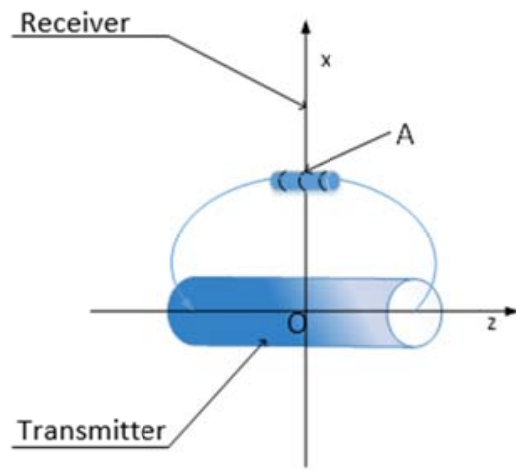

Fig. 4 Receiver Antenna Placement Schematic

\section{Hardware Demonstration Platform for Positioning System}

Pipeline detector positioning system is divided into three parts, namely, ultra-low frequency transmitter, receiver and data acquisition and processing part of the overall system block diagram shown in Figure 5.

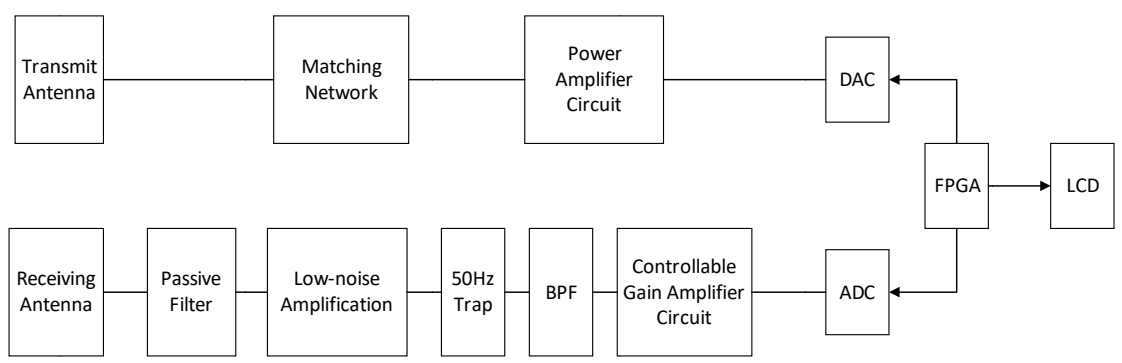

Fig. 5 System overall block diagram

\section{Conclusions}

In the laboratory, a simple magnetic antenna emission and reception experiment was carried out. The transmission distance was $1 \mathrm{~m}$, the coil current was $1 \mathrm{~A}$, the coil inductance was $2.2 \mathrm{mH}$, the resistance was $1 \Omega$, the emission frequency was $23 \mathrm{~Hz}$. The instrumentation was amplified, using the oscilloscope observed in the spectrum shown in Figure 6, you can clearly see the emission frequency spectrum. After the FFT in the FPGA after conditioning the signal processing, find the corresponding frequency of amplitude, to achieve positioning processing.

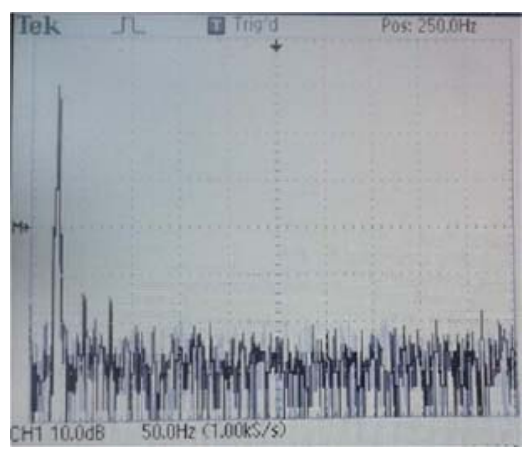

Fig. 6 Receiver Spectrum 


\section{Acknowledgments}

This research has been supported by International Science and Technology Cooperation Program of China (2014 DFR10240), Hei Long Jiang Postdoctoral Foundation (LBH-Z14066), Science Foundation of Heilongjiang Province QC2015075, and Fundamental Research Funds for the Central Universities GK2080260146.

\section{References}

[1] WU Xudong, HOU Wensheng, ZHENG Xiaolin, PENG Chenglin.Position Model and Experimental Verification of Magnetic Dipole [J] .Journal of Instrumentation, 2008,02: 326-329.

[2] GUO Jing-bo, CAI Xiong, HU Tie-hua, ZHANG Zhi-wen.Review on Key Techniques of Intelligent Robot Tracking and Positioning in Oil and Gas Pipeline [J] .Journal of Instrumentation, 2015,03: 481-498.

[3] WEI Mingsheng, TONG Minming, ZI Bin, XIA Jing, LU Yang.Radioelectromagnetic adaptive positioning technology of pipeline robot $[\mathrm{J}]$. Editorial Office of Optics and Precision Engineeri, 2012,04: 772-781.

[4] Li Junyuan. Based on ultra-low frequency electromagnetic wave of the pipeline robot tracer positioning technology research [D]. Harbin Institute of Technology, 2006.

[5]CHANG ChaoHsi. The magnetic dipole transitions in the binding system[J]. Science China(Physics,Mechanics \&amp; Astronomy),2010,11:2025-2030. 